\title{
Pathologies IV
}

\section{Citation}

Mumford, David B. 1975. Pathologies IV. American Journal of Mathematics 97(3): 847-849.

\section{Published Version}

doi: $10.2307 / 2373780$

\section{Permanent link}

http://nrs.harvard.edu/urn-3:HUL.InstRepos:3612775

\section{Terms of Use}

This article was downloaded from Harvard University's DASH repository, and is made available under the terms and conditions applicable to Other Posted Material, as set forth at http:// nrs.harvard.edu/urn-3:HUL.InstRepos:dash.current.terms-of-use\#LAA

\section{Share Your Story}

The Harvard community has made this article openly available.

Please share how this access benefits you. Submit a story.

\section{Accessibility}




\title{
PATHOLOGIES IV.
}

\author{
By David Mumford.
}

In this note I would like to use the beautifully simple method introduced by Tony Iarrobino [1] - when he proved that there are 0-dimensional subschemes of $\mathbf{P}^{3}$ which are not specializations of reduced subschemes-to prove here that there are also reduced and irreducible complete curves which are not specializations of non-singular curves. Since there are no global obstructions in deforming reduced curves, this also shows that there are complete reduced 1-dimensional local rings with no flat deformation which is generically smooth.

Start with a complete non-singular curve $C$ of genus $g$ with no automorphisms over an algebraically closed ground field $k$. Choose a point $x \in C$ and a large even integer $\nu$. Note that if $V$ is any $k$-vector space where

$$
m_{x, C}^{2 \nu} \subset V \subset m_{x, C}^{\nu}
$$

then $k+V$ is a subring of $\theta_{x, C}$. For each such $V$, define a new curve:

by:

$$
\pi: C \rightarrow C(V)
$$

(a) $\pi$ is a bijection, and an isomorphism

$$
\operatorname{res} \pi: C-\{x\} \stackrel{\approx}{\rightarrow} C(V)-\{\pi x\} .
$$

(b) $\theta_{\pi x, C(V)}=k+V$.

Note that if $V_{1}, V_{2}$ are two such vector spaces, then

$$
C\left(V_{1}\right) \approx C\left(V_{2}\right) \Rightarrow V_{1}=V_{2}
$$

(In fact, $C$ is the normalization of each $C(V)$; hence any $o: C\left(V_{1}\right) \stackrel{\approx}{\rightarrow} C\left(V_{2}\right)$ lifts to $o^{\prime}: C \rightarrow C$ which must be the identity, hence $k+V_{1}=\theta_{\pi(x), \mathrm{C}\left(V_{1}\right)}$ $=\vartheta_{\pi(x), C\left(V_{2}\right)}=k+V_{2}$.) Moreover, the curves $C(V)$ can all be fitted together into

Manuscript received December 14, 1973.

American Journal of Mathematics, Vol. 97, No. 3, pp. 847-849

Copyright (C) 1975 by Johns Hopkins University Press. 
a family if we fix the integer $\operatorname{dim}_{k} V / m_{x, C}^{2 \nu}$ : for all $k, 0 \leqslant k \leqslant \nu$,

let $G=$ Grassmanian of $k$-dimensional subspaces of $m_{x, C}^{\nu} / m_{x, C}^{2 \nu}$,

let $\mathscr{V} \subset\left(m_{x, C}^{\nu} / m_{x, C}^{2 \nu}\right) \otimes_{k} \vartheta_{G}$ be the universal family,

let $C(\mathscr{V})$ be the scheme equal to $C \times G$ as topological space, with structure sheaf defined by:

$$
\begin{aligned}
& \theta_{C \times G} \cdot \stackrel{\alpha}{\rightarrow} \quad\left(\theta_{x, C} / m_{x, C}^{2 \nu}\right) \otimes_{k} \theta_{G} \quad \longrightarrow 0 \\
& \text { U } \\
& \cup \quad\left[k+\left(m_{x, C}^{\nu} / m_{x, C}^{2 \nu}\right)\right] \otimes_{k} \theta_{G} \\
& \cup \\
& \alpha^{-1}\left(\mathfrak{V}+\theta_{G}\right) \quad---\rightarrow \quad \mathfrak{V}+\theta_{G} \\
& \text { \|def. } \\
& \theta_{C(n)}
\end{aligned}
$$

Since $\left[\left(\vartheta_{x, C} / m_{x, C}^{2 \nu}\right) \otimes_{k} \theta_{G}\right] / \mathscr{V}$ is a locally free $\theta_{G}$-sheaf, $\theta_{C(\vartheta)}$ is flat over $\theta_{G}$, i.e., $C(\mathscr{V})$ is flat over $G$.

Now choose $k=\nu / 2$ and calculate:

(i) $\operatorname{dim} G=k(\nu-k)=\nu^{2} / 4$

(ii) $\quad p_{a}(C(V))=g+\operatorname{dim}_{k}\left[\Theta_{x, C} / \mathcal{\Theta}_{\pi(x), C(V)}\right]$

$$
=g+\left(\frac{3 \nu}{2}-1\right)
$$

Therefore if $\nu \gg 0, \operatorname{dim} G \geqslant 3 p_{a}(C(V))-3$ ! I claim that this implies that almost all the curves $C(V)$ are not specializations of non-singular curves, because of:

Lemma. Let $p: \Theta \rightarrow S$ be a flat and proper family of reduced and irreducible singular curves $C_{s}=p^{-1}(s)$ such that

(a) $\forall s \in S,\left\{s^{\prime} \mid C_{s^{\prime}} \approx C_{s}\right\}$ is finite

(b) $p_{a}\left(C_{s}\right) \geqslant 2, S$ is irreducible and $\operatorname{dim} S \geqslant 3 p_{a}\left(C_{s}\right)-3$,

then almost all curves $C_{s}$ are not specializations of non-singular curves.

Proof. If the conclusion is false, then after replacing $S$ by a Zariski open subset we can extend the family $C / S$ like this:

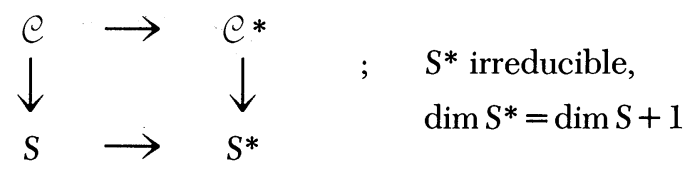


so that $C^{*}$ is generically smooth over $S^{*}$. In fact $C$ will carry a relatively ample $L$, so we may use $p_{*} L^{\otimes n}(n \gg 0)$ to embed $\bigodot$ in some $\mathbf{P}^{N}$-bundle $\mathscr{P}$ over $S$. Moreover, if a $C_{S}\left(s \in S_{0}\right)$ is abstractly a specialization of a non-singular curve, so is the embedded curve $C_{S} \subset \mathbf{P}^{N}$. So take $S^{*}$ to be a suitable subvariety of the Hilbert scheme of $\mathcal{P}$ over $S$. Once we have $\mathcal{C}^{*} / S^{*}$, consider the two induced families:

$$
\mathcal{C}_{i}^{*}=C^{*} \times{ }_{S *}\left(S^{*} \times S^{*}\right) \quad\left(\text { formed via } p_{i}: S^{*} \times S^{*} \rightarrow S^{*}, i=1,2\right)
$$

and the scheme

$$
I=\operatorname{Isom}_{S^{*} \times S^{*}}\left(\bigodot_{1}^{*}, \bigodot_{2}^{*}\right)
$$

whose points over $\left(s_{1}, s_{2}\right) \in S^{*} \times S^{*}$ are isomorphisms $o: C_{s_{1}} \rightarrow C_{s_{2}}$. Look at the morphisms:

$$
\begin{array}{lll} 
& \left.\begin{array}{l}
I \\
\downarrow
\end{array}\right) & q\left(o: C_{s_{1}} \rightarrow C_{s_{2}}\right)=s_{1} \\
S^{*} & \delta(s)=\left[\text { id. }: C_{s} \rightarrow C_{s}\right]
\end{array}
$$

Since $\operatorname{dim} S^{*}=\operatorname{dim} S+1>3 p_{a}\left(C_{s}\right)-3$, whenever $C_{s}$ is non-singular, the same non-singular curve must occur in the family $C^{*} / S^{*}$ infinitely often; thus when $C_{s}$ is non-singular, some component of $q^{-1}(s)$ through $\delta(s)$ is positivedimensional. Now by upper semi-continuity of dimensions of fibres of a morphism, it follows that for every $s, q^{-1}(s)$ has a positive-dimensional component through $\delta(s)$. Now let $D_{1}=\operatorname{Im}\left(S \rightarrow S^{*}\right), D_{2}=\left\{s \mid C_{s}\right.$ is singular $\}$; then $\bar{D}_{1}$ is a component of $D_{2}$ and let $D_{1}^{0}=D_{1}$-(closure of $\left.D_{2}-D_{1}\right)$. Choose $s \in D_{1}^{0}$ and consider how $q^{-1}(s)$ can have a positive-dimensional component $\gamma$ through $\delta(s)$. By (b), Aut $\left(C_{s}\right)$ is finite; by (a), there are only finitely many $s^{\prime} \in D_{1}$ with $C_{s} \approx C_{s^{\prime}}$; certainly $C_{s} \approx C_{s^{\prime}}$ if $s^{\prime} \in S^{*}-D_{2}$ because $C_{s}$ is singular while $C_{s^{\prime}}$ is non-singular; and since $s \notin$ (closure $\left.D_{2}-D_{1}\right), \gamma$ cannot lie over $(s) \times \overline{D_{2}-D_{1}}$ in $S^{*} \times S^{*}$. Thus there is nowhere for $\gamma$ to go! Contradiction.

\section{REFERENCES.}

[1] A. Iarrobino, "Reducibility of the families of 0-dimensional schemes on a variety," Inv. Math. 15 (1972), pp. 72-77. 